\title{
Top-Down Characterization of the Post-Translationally Modified Intact Periplasmic Proteome from the Bacterium Novosphingobium aromaticivorans
}

\author{
Si Wu, ${ }^{1}$ Roslyn N. Brown, ${ }^{2}$ Samuel H. Payne, ${ }^{3}$ Da Meng, ${ }^{4}$ Rui Zhao, ${ }^{1}$ \\ Nikola Tolić, ${ }^{1}$ Li Cao, ${ }^{5}$ Anil Shukla, ${ }^{3}$ Matthew E. Monroe, ${ }^{3}$ Ronald J. Moore, ${ }^{3}$ \\ Mary S. Lipton, ${ }^{3}$ and Ljiljana Paša-Tolić ${ }^{1}$ \\ ${ }^{1}$ Environmental Molecular Science Laboratory, Pacific Northwest National Laboratory, P.O. Box 999/MS K8-98, Richland, \\ WA 99352, USA \\ ${ }^{2}$ Center for Bioproducts and Bioenergy, Washington State University, Richland, WA, USA \\ ${ }^{3}$ Biological Sciences Division, Pacific Northwest National Laboratory, Richland, WA, USA \\ ${ }^{4}$ Computational Sciences and Mathematics Division, Pacific Northwest National Laboratory, Richland, WA, USA \\ ${ }^{5}$ Department of Neurobiology, 720 Westview Drive SW, Atlanta, GA, USA
}

Correspondence should be addressed to Ljiljana Paša-Tolić; ljiljana.pasatolic@pnnl.gov

Received 7 October 2012; Revised 31 January 2013; Accepted 4 February 2013

Academic Editor: Boris Zybailov

Copyright (C) 2013 Si Wu et al. This is an open access article distributed under the Creative Commons Attribution License, which permits unrestricted use, distribution, and reproduction in any medium, provided the original work is properly cited.

\begin{abstract}
The periplasm of Gram-negative bacteria is a dynamic and physiologically important subcellular compartment where the constant exposure to potential environmental insults amplifies the need for proper protein folding and modifications. Top-down proteomics analysis of the periplasmic fraction at the intact protein level provides unrestricted characterization and annotation of the periplasmic proteome, including the post-translational modifications (PTMs) on these proteins. Here, we used single-dimension ultra-high pressure liquid chromatography coupled with the Fourier transform mass spectrometry (FTMS) to investigate the intact periplasmic proteome of Novosphingobium aromaticivorans. Our top-down analysis provided the confident identification of 55 proteins in the periplasm and characterized their PTMs including signal peptide removal, N-terminal methionine excision, acetylation, glutathionylation, pyroglutamate, and disulfide bond formation. This study provides the first experimental evidence for the expression and periplasmic localization of many hypothetical and uncharacterized proteins and the first unrestrictive, largescale data on PTMs in the bacterial periplasm.
\end{abstract}

\section{Introduction}

The periplasm of Gram-negative bacteria is a hydrated gel located between the cytoplasmic and outer membranes and is comprised of peptidoglycan (cell wall), proteins, carbohydrates, and small solutes [1-3]. The periplasm is a dynamic subcellular compartment important for trafficking of molecules into and out of cells, maintaining cellular osmotic balance, envelope structure, responding to environmental cues and stresses, electron transport, xenobiotic metabolism, and protein folding and modification [4].
The periplasm provides a good model system to study protein biogenesis, composition, sorting, and modification at the molecular level. Indeed, it is analogous in many ways to the endoplasmic reticulum of eukaryotic cells in terms of transport, folding, and quality control [3]. Localization to the periplasm and beyond often involves an N-terminal secretion signal that targets the protein for translocation across the cytoplasmic membrane via the general secretory pathway [5]. These secretion signals (also known as signal peptides) are cleaved by signal peptidases located in the cytoplasmic membrane [6]. Thus, it is expected that signal 
peptide cleavage is a common modification in the periplasmic proteome.

Compared to the cytoplasm, the periplasm is more vulnerable to changes in $\mathrm{pH}$, temperature, and osmolarity in the external environment $[4,7,8]$. For structural stability in diverse and dynamic environmental conditions, periplasmic proteins often contain disulfide bonds and the periplasm is maintained in an oxidizing state to facilitate this process $[9,10]$. Other PTMs, such as the addition of heme groups to cytochromes, may occur in the periplasm [11]. Therefore, the detailed study of bacterial periplasmic proteins not only allows for a better understanding of the physiology of microbial systems, but also provides information toward the complete annotation of mature proteoforms of microbial genomes, and it may give insight into protein sorting and PTMs in more complex systems.

The typical proteomic approach to profile the periplasm is the bottom-up liquid chromatography-tandem mass spectrometry (LC-MS/MS) approach [12,13]. It has been applied to the periplasmic proteome of the extremophile Acidithiobacillus ferrooxidans, where it yielded a total of 131 proteins [14]. A majority of the identified proteins in $A$. ferrooxidans were categorized as periplasmic proteins based on their predicted export signals using software such as SignalP. However, direct evidence for signal peptide removal such as $N$-terminal peptide identifications was not available in that study. The most significant drawback of the bottom-up approach is that it rarely provides complete sequence coverage to ensure the identification of $N$-terminal peptides, which can be essential for understanding localization. Moreover, when multiple PTMs occur in a single protein, the bottom-up approach cannot accurately define proteoforms, as it does not have the ability to determine which combinations of PTMs cooccur in a single proteoform.

To overcome these difficulties, we employ top-down mass spectrometry (MS) to study the periplasmic proteome. Topdown MS measures intact proteins and facilitates the full characterization of proteoforms including PTMs [15]. The top-down approach has been successfully applied for the characterization of various protein PTMs including signal peptide identification [16]. Recent improvements in intact protein LC separations and high performance FTMS instrumentation greatly expand the observable range of proteoforms. Because top-down analysis preserves the mature $\mathrm{N}$ terminus, the proteolytic processing (e.g., N-terminal cleavage) of a protein is evident. Thus top-down MS provides an experimental validation of bioinformatic predictions such as the signal peptide cleavage predicted by SignalP.

As an initial subject for analysis, we focused on the Gramnegative alphaproteobacterium, Novosphingobium aromaticivorans. Members of this genus are noted for their remarkable ability to degrade a variety of aromatic hydrocarbons [17]. The genome of only one species, $N$. aromaticivorans, has been completely sequenced. In a genome with 3917 proteins, nearly 30\% are annotated as "hypothetical"; moreover, using the subcellular localization predictor PSORTb (http://www.psort.org/psortb/), 33\% of proteins have "unknown" localization. Our current goal is to identify protein constituents of the periplasm of this unusual microor- ganism, to aid in annotation of hypothetical and poorly characterized proteins, and to survey, in an unrestricted manner, the PTMs existing in these proteins. We here report our results on profiling the enriched periplasmic proteome of $N$. aromaticivorans using a high throughput intact protein (top-down) analysis. A total of 55 proteins were confidently identified, and their PTMs were characterized including Nterminal processing (e.g., signal peptide removal), acetylation, glutathionylation, pyroglutamate modification, and disulfide bond formation.

\section{Experimental Section}

2.1. Periplasmic Protein Extraction. N. aromaticivorans str. DSM 12444 was grown to early stationary phase aerobically in $50 \%(\mathrm{v} / \mathrm{v})$ Luria Bertani broth. Cells were harvested by centrifugation at $8,500 \mathrm{~g}$ for $5 \mathrm{~min}$, washed once with sodium phosphate ( $\mathrm{pH} 7.5$ ), and the periplasm extracted as previously reported [18]. The soluble periplasmic fraction was flashfrozen in liquid nitrogen and concentrated using a SpeedVac (Thermo-Savant) prior to top-down analysis. For peptidelevel analysis, 4 volumes of $20 \%$ acetonitrile and 3 volumes of water were added, followed by trypsin (trypsin to protein ratio $1: 50$ ), and incubated at $37^{\circ} \mathrm{C}$ for $18 \mathrm{~h}$. The sample was concentrated to dryness in a SpeedVac and suspended in $20 \mu \mathrm{L} 0.1 \%$ formic acid prior to LC-MS/MS analysis.

2.2. Intact Protein LC-MS/MS Analysis. The intact protein RPLC separation was performed on a Waters NanoAcquity system with a column $(80 \mathrm{~cm} \times 75 \mu \mathrm{m}$ i.d. $)$ packed in-house with Phenomenex Jupiter particles (C5 stationary phase, $5 \mu \mathrm{m}$ particle size, $300 \AA$ pore size). Mobile phase A was composed of $0.5 \%$ acetic acid, $0.01 \%$ TFA, $5 \%$ isopropanol, $10 \%$ acetonitrile $(\mathrm{ACN})$, and $84.5 \%$ water. Mobile phase $\mathrm{B}$ consisted of $0.5 \%$ acetic acid, $0.01 \%$ TFA, $9.9 \%$ water, $45 \%$ isopropanol, and $45 \% \mathrm{ACN}$. The operating flow rate was $0.3 \mu \mathrm{L} / \mathrm{min}$. The RPLC system was equilibrated with $100 \%$ mobile phase A for 5 minutes and then increased to $20 \%$ mobile phase B in 1 minute. A 250 minute linear gradient was set from $20 \%$ mobile phase B to $55 \%$ mobile phase $B$. MS analysis was performed using an LTQ-Orbitrap Velos spectrometer (Thermo Scientific, San Jose, CA) outfitted with a custom electrospray ionization (ESI) interface. ESI emitters were custom made using 150 um o.d. $\times 20$ um i.d. chemically etched fused silica [19]. The heated capillary temperature and spray voltage were $275^{\circ} \mathrm{C}$ and $2.2 \mathrm{kV}$, respectively. Two LC-MS/MS analyses were performed: one with ETD fragmentation and one with HCD fragmentation. For the LCMS/MS analysis with ETD fragmentation, a parent spectrum was collected at a $60 \mathrm{~K}$ resolution and was followed by high resolution $(30 \mathrm{~K}) \mathrm{ETD} \mathrm{MS} / \mathrm{MS}$ of the 8 most intense ions from the parent spectrum. The ETD reaction time was fixed at $40 \mathrm{~ms}$. For the LC-MS/MS analysis with HCD fragmentation, a parent spectrum was collected at a $60 \mathrm{~K}$ resolution and was followed by high resolution $(30 \mathrm{~K}) \mathrm{HCD}$ MS/MS of the 8 most intense ions from the parent spectrum. FT MS/MS employed $45 \%$ normalized collision energy for HCD. Mass calibration 
TABLE 1: Modifications of identified proteins using top-down approach.

\begin{tabular}{|c|c|c|c|c|c|}
\hline Locus_Tag & Genbank Protein Desc & Export signal & $\begin{array}{c}\text { Detected } \\
\text { signal peptide }^{\mathrm{a}}\end{array}$ & N-terminal & Other modifications \\
\hline Saro_2004 & $\begin{array}{l}\text { Alkyl hydroperoxide reductase/Thiol } \\
\text { specific antioxidant/Mal allergen }\end{array}$ & $\operatorname{Sec} P$ & None & Removal of met & \\
\hline Saro_2586 & Cold-shock DNA-binding protein family & SecP & None & Removal of met & \\
\hline Saro_0565 & Glutathione peroxidase & SecP & None & Removal of met & \\
\hline Saro_0483 & Superoxide dismutase & $\operatorname{SecP}$ & None & Removal of met & \\
\hline Saro_3290 & Thiamine biosynthesis protein ThiS & SecP & None & Removal of met & \\
\hline Saro_1996 & Thioredoxin & SecP & None & Removal of met & Disulfide bond \\
\hline Saro_1332 & CsbD-like protein & SecP/TatP & None & Removal of met & \\
\hline Saro_1919 & Hypothetical protein & SecP/TatP & Unknown & Proteolytic fragment & \\
\hline Saro_1314 & Conserved hypothetical protein & SecP/TatP & Yes & AXA & Disulfide bond, pyro glu \\
\hline Saro_2385 & Hypothetical protein & SecP/TatP & Yes & AXA & Pyro glu \\
\hline Saro_3257 & Conserved hypothetical protein & SignalP & None & Proteolytic fragment & \\
\hline Saro_3518 & Cupin 2, conserved barrel domain protein & SignalP & None & $\begin{array}{c}\text { Removal of met } \\
\text { (wrong starting site) }\end{array}$ & \\
\hline Saro_3173 & OmpA/MotB & SignalP & None & Proteolytic fragment & \\
\hline Saro_1303 & Hypothetical protein & SignalP & Unknown & Proteolytic fragment & \\
\hline Saro_1685 & Amine dehydrogenase & SignalP & Yes & AXA & \\
\hline Saro_2852 & Ankyrin & SignalP & Yes & AXA & Pyro glu \\
\hline Saro_3053 & Beta-Ig-H3/fasciclin & SignalP & Yes & AXA & \\
\hline Saro_0830 & Cell wall surface anchor family protein & SignalP & Yes & VAA, not AXA & \\
\hline Saro_2955 & Conserved hypothetical protein & SignalP & Yes & AXA & \\
\hline Saro_1378 & Conserved hypothetical protein & SignalP & Yes & AXA & \\
\hline Saro_0103 & Conserved hypothetical protein & SignalP & Yes & AXA & Disulfide bond \\
\hline Saro_2067 & Conserved hypothetical protein & SignalP & Yes & SHA, not AXA & Pyro glu \\
\hline Saro_1721 & Conserved hypothetical protein & SignalP & Yes & THA, not AXA & \\
\hline Saro_2522 & Hypothetical protein & SignalP & Yes & ASN, not AXA & Disulfide bond \\
\hline Saro_3326 & Hypothetical protein & SignalP & Yes & AXA & Disulfide bond \\
\hline Saro_2384 & Hypothetical protein & SignalP & Yes & AXA & Pyro glu \\
\hline Saro_1978 & Hypothetical protein & SignalP & Yes & AXA & Pyro glu \\
\hline Saro_1502 & Hypothetical protein & SignalP & Yes & AXA & \\
\hline Saro_1412 & Hypothetical protein & SignalP & Yes & AXA & Disulfide bond \\
\hline Saro_2350 & Peptidase M28 & SignalP & Yes & AXA & \\
\hline Saro_0837 & $\begin{array}{l}\text { Peptidyl-prolyl cis-trans isomerase, } \\
\text { cyclophilin type }\end{array}$ & SignalP & Yes & LVA, not AXA & \\
\hline Saro_2251 & Peptidylprolyl isomerase & SignalP & Yes & VAA, not AXA & Pyro glu \\
\hline Saro_0989 & Peptidylprolyl isomerase, FKBP-type & SignalP & Yes & AIS, not AXA & Disulfide bond \\
\hline Saro_0823 & Protein of unknown function DUF192 & SignalP & Yes & AXA & \\
\hline Saro_3075 & TonB-dependent receptor & SignalP & Yes & AXA & Proteolytic fragment \\
\hline Saro_2265 & YceI & SignalP & Yes & MVA, not AXA & Pyro glu \\
\hline Saro_1171 & Hypothetical protein & SignalP/SecP & None & Removal of met & Disulfide bond \\
\hline Saro_1420 & Antibiotic biosynthesis monooxygenase & TatP & None & Removal of met & Disulfide bond \\
\hline Saro_3279 & Arsenate reductase & TatP & None & N/A & $\begin{array}{l}\text { S-glutathiolation on } \\
\text { cysteine }\end{array}$ \\
\hline
\end{tabular}


TABle 1: Continued.

\begin{tabular}{|c|c|c|c|c|c|}
\hline Locus_Tag & Genbank Protein Desc & Export signal & $\begin{array}{c}\text { Detected } \\
\text { signal peptide }^{\mathrm{a}}\end{array}$ & N-terminal & Other modifications \\
\hline Saro_1703 & (2Fe-2S)-binding protein & & None & Removal of met & Disulfide bond \\
\hline Saro_1346 & (2Fe-2S)-binding protein & & None & Removal of met & Disulfide bond \\
\hline Saro_1339 & Acyl carrier protein & & None & Removal of met & Modification (382 Da) \\
\hline Saro_2520 & BolA-like protein & & None & Removal of met & \\
\hline Saro_0034 & Chaperonin Cpn10 & & None & Removal of met & \\
\hline Saro_2299 & Conserved hypothetical protein & & None & Removal of met & \\
\hline Saro_2229 & GreA/GreB family elongation factor & & None & Removal of met & Both acetylation and $\mathrm{n} / \mathrm{a}$ \\
\hline Saro_2403 & $\begin{array}{l}\text { H+-transporting two-sector ATPase, } \\
\text { delta/epsilon subunit }\end{array}$ & & None & Removal of met & \\
\hline Saro_1768 & Hypothetical protein & & None & Removal of met & \\
\hline Saro_1177 & Hypothetical protein & & None & Removal of met & \\
\hline Saro_1778 & Molybdopterin binding domain & & None & Removal of met & $\begin{array}{l}\text { S-glutathiolation on } \\
\text { cysteine }\end{array}$ \\
\hline Saro_0894 & Nucleoside diphosphate kinase & & None & Removal of met & \\
\hline Saro_1830 & PhnA protein & & None & Removal of met & \\
\hline Saro_1033 & $\begin{array}{l}\text { Phosphoribosylformylglycinamidine } \\
\text { synthetase PurS }\end{array}$ & & None & $\mathrm{N} / \mathrm{A}$ & \\
\hline Saro_0209 & Tetratricopeptide TPR_4 & & None & $\begin{array}{l}\text { Removal of met } \\
\text { (wrong starting site) }\end{array}$ & Pyro glu \\
\hline Saro_1194 & Hypothetical protein & & Yes & $\mathrm{AXA}$, unusual ${ }^{\mathrm{b}}$ & Disulfide bond \\
\hline
\end{tabular}

${ }^{\text {a }}$ Signal peptide cleavage was annotated as described in the Experimental section.

${ }^{\mathrm{b}}$ The unusual cleavage site was further validated. See discussion in text and Figure 3.

was performed prior to analysis according to the method recommended by the instrument manufacturer.

2.3. Capillary LC-MS/MS Analysis on Trypsin-Digested Peptides. Bottom-up identification of proteins was achieved through the detection of peptides with LC-MS/MS. The capillary RPLC system used for peptide separations has been previously described [20]. Briefly, the HPLC system consisted of a custom configuration of $100 \mathrm{~mL}$ ISCO Model 100DM syringe pumps (Isco, Inc., Lincoln, NE), 2-position Valco valves (Valco Instruments Co., Houston, TX), and a PAL autosampler (Leap Technologies, Carrboro, NC), allowing for fully automated sample analysis across four separate HPLC columns ( $3 \mu \mathrm{m}$ Jupiter C18 stationary phase, Phenomenex, Torrance, CA). Mobile phases consisted of $0.1 \%$ formic acid in water (A) and $0.1 \%$ formic acid acetonitrile (B). The HPLC system was equilibrated at $10 \mathrm{kpsi}$ with $100 \%$ mobile phase A, and a mobile phase selection valve was switched $50 \mathrm{~min}$ after injection, which created a near-exponential gradient as mobile phase $\mathrm{B}$ displaced $\mathrm{A}$ in a $2.5 \mathrm{~mL}$ active mixer. A $40 \mathrm{~cm}$ length of $360 \mu \mathrm{m}$ o.d. $\times 15 \mu \mathrm{m}$ i.d. fused silica tubing was used to split $\sim 17 \mu \mathrm{L} / \mathrm{min}$ of flow before it reached the injection valve ( $5 \mu \mathrm{L}$ sample loop). The split flow controlled the gradient speed under conditions of constant pressure operation (10 kpsi). Flow through the capillary HPLC column when equilibrated to $100 \%$ mobile phase A was $~ 500 \mathrm{~nL} / \mathrm{min}$. ESI using an etched fused-silica tip [19] was employed to interface the RPLC separation to an LTQ mass spectrometer (Thermo Scientific, San Jose, CA). Precursor ion mass spectra (automatic gain control was set to $1 \times 10^{6}$ ) were collected for $400-2000 \mathrm{~m} / \mathrm{z}$ range at a resolution of $100 \mathrm{~K}$ followed by data dependent ion trap CID MS/MS (collision energy 35\%, AGC $3 \times 10^{4}$ ) of the ten most abundant ions. A dynamic exclusion time of $180 \mathrm{sec}$ was used to discriminate against previously analyzed ions.

2.4. Data Analysis. Intact protein MS/MS data were subjected to data analysis and protein identification using MSAlign $+{ }^{16}$ (http://bix.ucsd.edu/projects/msalign/) with the following search parameters: minimal precursor mass = $2500 \mathrm{Da}$; minimal fragment peaks per scan $=10$; maximum number of modifications $=2$; fragment mass error tolerance $=15 \mathrm{ppm}$. MS-Align+ reported only the PrSM with the best E-value for each spectrum. LC-MS/MS data were searched against the Genbank protein annotation (accession CP000248). The false discovery rate (FDR) for protein/spectrum matches was estimated by searching all top-down spectra against the human Uniprot database. A final cutoff of E-value $2.7 \mathrm{E}^{-4}$ was used to achieve FDR 1\%. Protein identifications were further manually verified. Peptide-level MS/MS data were searched using SEQUEST and were filtered using MSGF [21] with a spectral probability cutoff of $1 \mathrm{E}^{-10}$. All the raw datasets and MSAlign+ output results were deposited at http://omics.pnl .gov/view/publication_1074.html.

Signal peptides were determined using the identified peptides and the prokaryotic proteogenomic pipeline [22]. 


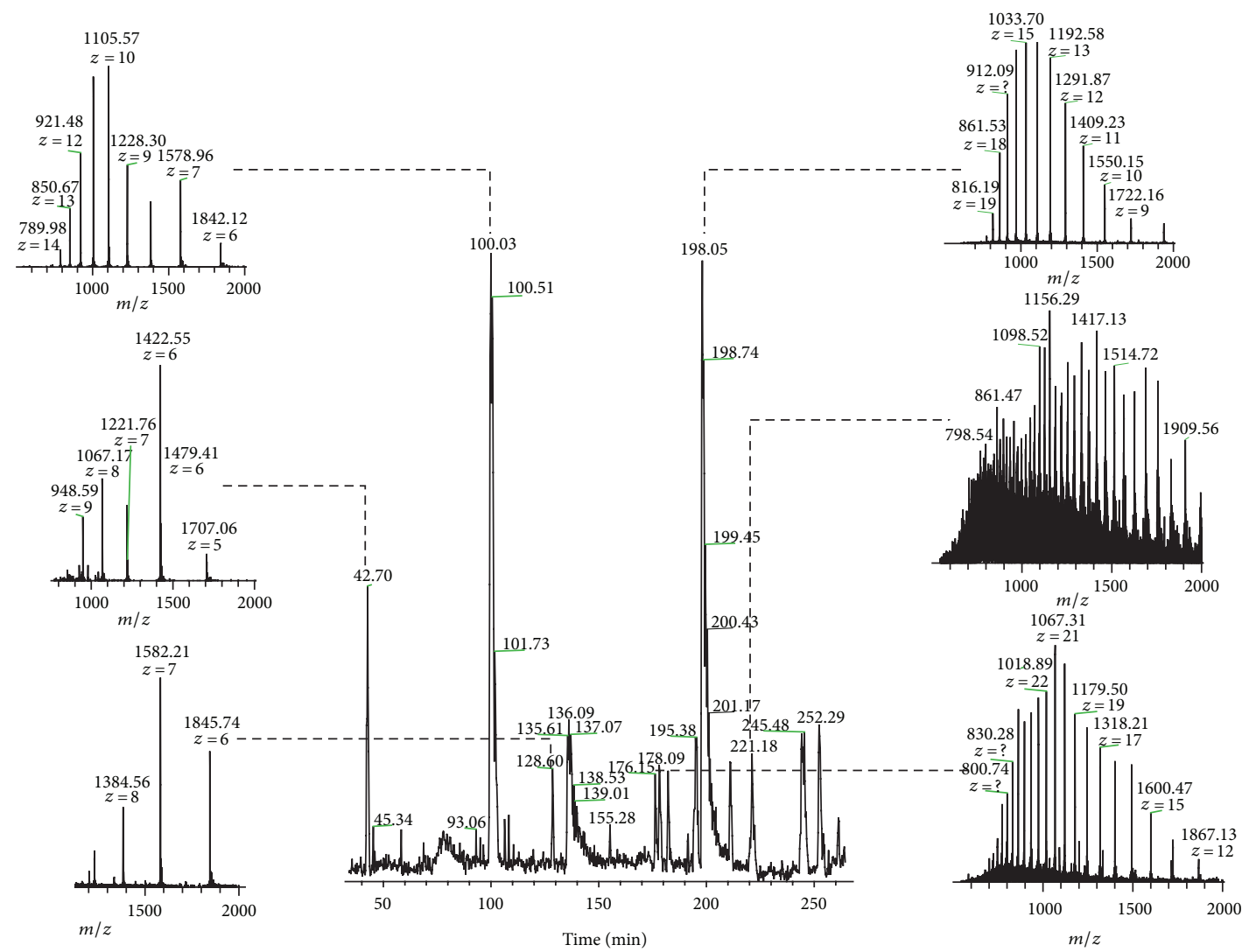

FIGURE 1: Total ion chromatogram (TIC) of an RPLC-MS analysis of intact periplasmic protein from N. aromaticivorans. Several representative intact protein spectra are highlighted.

The three criteria were taken from previously recognized signal peptide characteristics [23]. We required a hydrophobic patch of at least eight contiguous amino acids and examined the signal peptide C-terminus for the expected A-X-B cleavage motif (where $\mathrm{A}=$ [Ile, Val, Leu, Ala, Gly, Ser, Thr], X $=$ any amino acid, $\mathrm{B}=$ [Ala, Gly, Ser $]$ ). We also required a basic residue between the start and the hydrophobic patch. Typically the mature protein starts between 15 and 35 residues from the initiator methionine. However, due to the possibility of incorrectly annotated start sites, we allowed for some variance from this requirement.

Subcellular localization and protein functional predictions were made using PSORTb (http://db.psort.org/ browse/genome?id=8602), SignalP (http://www.cbs.dtu.dk/ services/SignalP/), SecretomeP (http://www.cbs.dtu.dk/services/SecretomeP/), and the Comprehensive Microbial Resource Genome Tools (http://cmr.jcvi.org/tigr-scripts/CMR/ shared/Genomes.cgi).

\section{Results and Discussion}

To study the mature proteoforms and PTMs of the $N$. aromaticivorans periplasm, the periplasmic fraction was prepared as previously reported [18]. The enriched intact periplasmic protein fraction was subjected to nano-LCMS/MS using two different fragmentation methods. Figure 1 shows the base peak chromatogram of a 300-minute LC-MS analysis with several representative intact protein spectra. The detected protein masses varied from $4 \mathrm{kDa}$ to $40 \mathrm{kDa}$. Intact MS/MS data were analyzed using MS-Align+ [15]. In total, 55 proteins were identified at a $1 \%$ FDR (Table 1).

To highlight the specificity and efficiency of the enrichment, we note that abundant cytoplasmic proteins were largely absent in the periplasmic preparation, indicating a low amount of cell lysis during the experiments. For example, none of the ribosomal proteins were detected. Small, highly abundant cytoplasmic proteins such as ribosomal proteins typically dominate in global (whole cell) top-down LC-MS analyses and are often detected in membrane fractions [24, 25]. Several of the proteins identified here were expected to be localized to the periplasm. For example, superoxide dismutase (Saro_0483), a tetratricopeptide repeat protein (Saro_0209), and two peptidyl-prolyl cis-trans isomerases (Saro_0837 and Saro_2251) are known to be localized in the periplasm in other Gram-negative bacteria. Moreover, these proteins were predicted by PSORTb to be periplasmic in $N$. aromaticivorans, and the latter three were enriched in the periplasm of this organism, compared to the cytoplasm, inner membrane, or outer membrane fractions, in a proteomic analysis of multiple subcellular fractions (data not shown). Also, it should be noted that some outer membrane proteins have periplasmic domains. For example, half of 


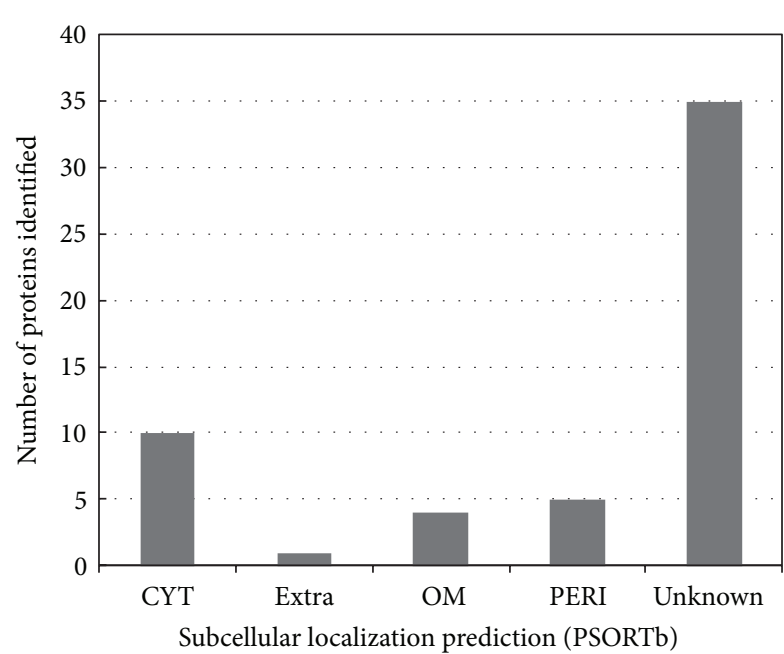

(a)

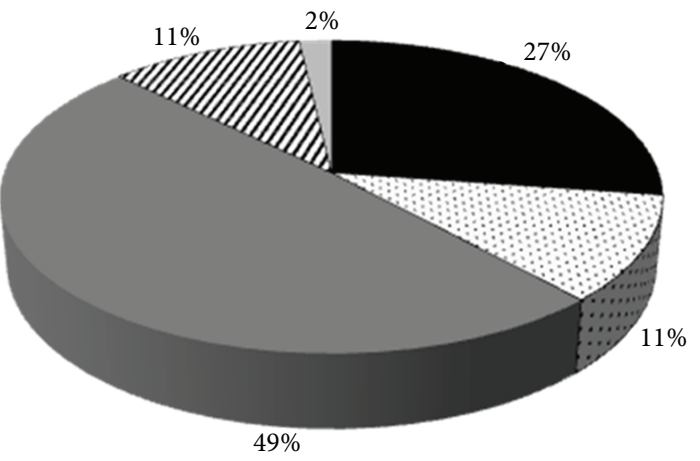

Predicted export signal

SecP Other

SignalP

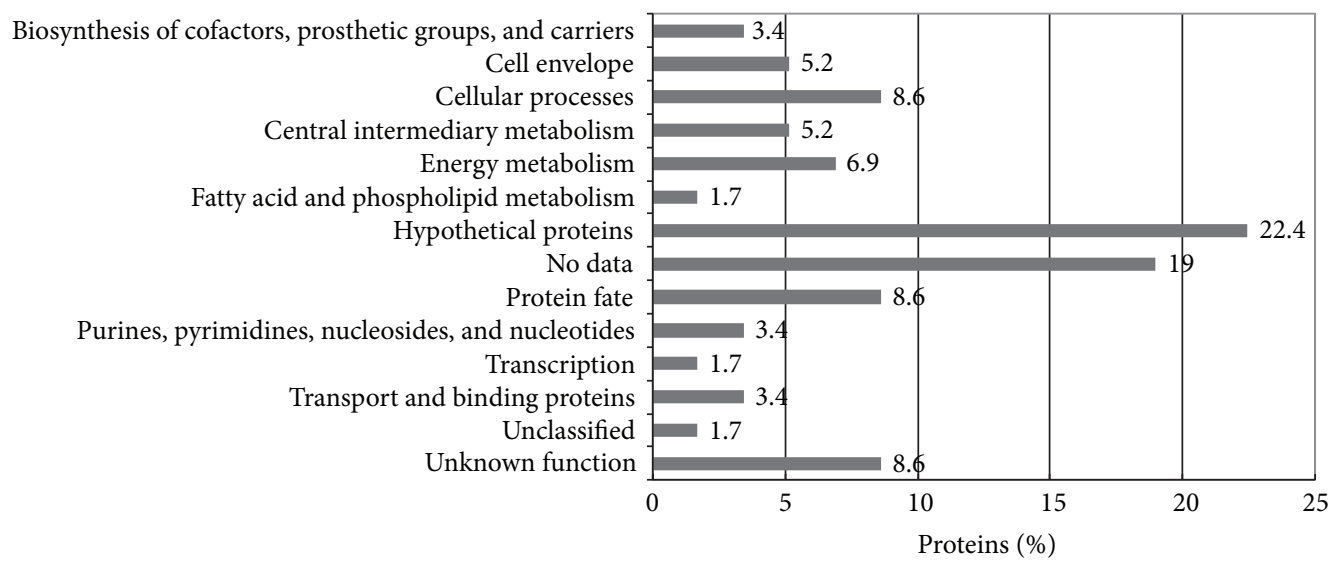

(c)
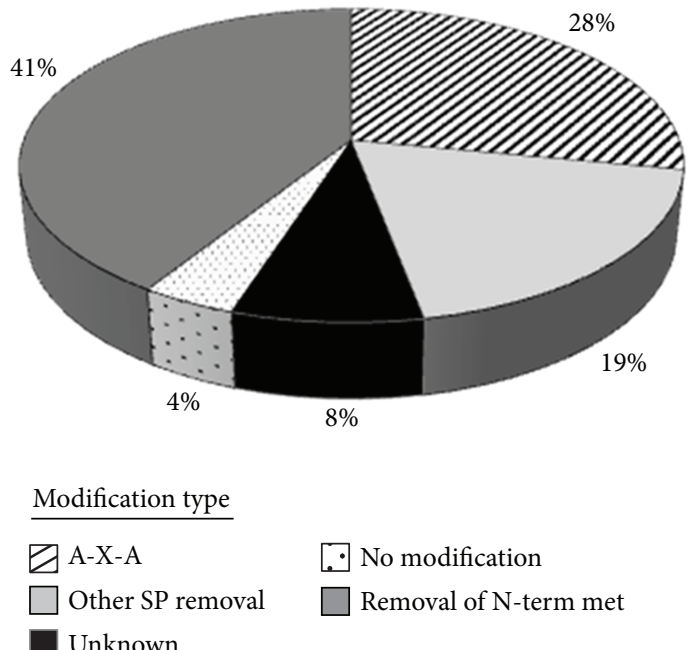

(d)

FIGURE 2: (a) Subcellular localization prediction using PSORTb: cytoplasmic (CYT), inner membrane (IM), extracellular (EXTRA), outer membrane (OM), and periplasmic (PERI). (b) Pie chart showing the distribution of predicted export signals among proteins identified by top-down MS. One predicted PERI protein with no detected export signal is designated as "Other." (c) JCVI annotated functional categories of intact proteins identified using top-down analysis. (d) Pie chart representation of protein modifications observed by top-down MS. 
Hypothetical protein Saro_1194

$E$-value: $1.4 E-27$; total number of matched peaks: 32

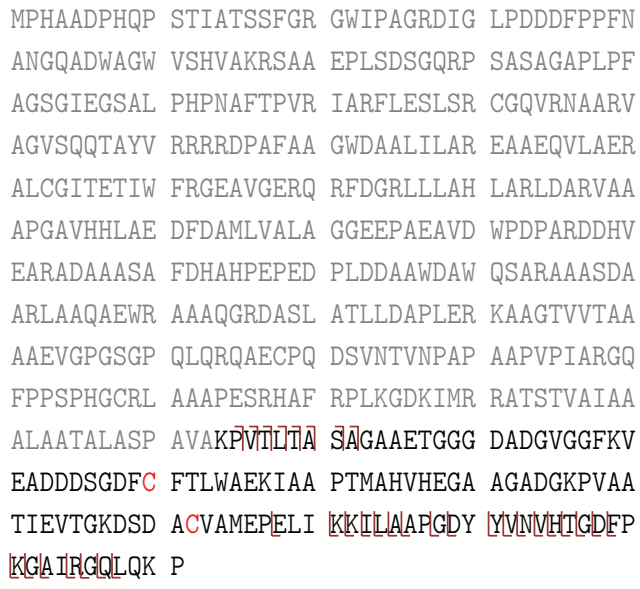

FIGURE 3: Fragmentation ion map of the uncharacterized protein Saro_1194 using intact protein MS/MS, indicating that the mature protein contains only the C-terminal portion of the predicted protein sequences starting at residue 414 (the portion of the sequence labeled with grey font was not detected in this experiment).

OmpA (residues 172-325) is periplasmic [26], resulting in its identification in the periplasm here. Subcellular localization predictions of the identified proteins are shown in Figure 2(a). The majority fall into the "unknown" category, making this the first experimental data on subcellular localization for these proteins.

The first and most prevalent type of PTM identified via the top-down approach was proteolytic cleavage. The cleavage events described later were found to be uniformly present; there were no uncleaved forms of the protein detected. We categorized identified proteoforms according to known types of proteolytic maturation. Based on the observed signal peptide cleavage, 25 proteins were localized to the periplasm via Sec-dependent secretion (Figure 2(b)) with detected signal peptide removal. Upstream of the mature protein, the three hallmarks of signal peptides were clearly present: early basic residue(s), a hydrophobic patch of at least 8 residues, and the signal peptidase I cleavage motif. Sixteen proteins were detected with the predominant Ala-Xxx-Ala motif, while 7 of them exhibited tolerated variability at the -3 position [23]. Many of these proteins had poor functional characterization, with 21 lacking any functional annotation (Figure 2(c)) or significant match to protein domain descriptors (e.g., CDD or Pfam; Table 1). Thus, by identifying both their cellular location (periplasm) and their maturation processing, we have significantly added to the annotation of these proteins. We compared the observed signal peptide cleavage to the computational predictions from SignalP4.0 (Figure 2(b)). SignalP correctly predicted 23 of the 25 proteins as containing a signal peptide but did not determine the correct site of cleavage in 6 of the 23 (Table 1) based on topdown analysis. Moreover, SignalP had two false predictions where the identified proteoform lacked a cleaved signal peptide. Other computational tools were also applied such as TatP and SecP, yielding six more proteins with predicted export signals. Among these six proteins, only two were confirmed with cleaved signal peptides through top-down analysis. Therefore, top-down analysis provided additional information for confident protein categorization, which can be potentially incorporated with currently available software tools to further improve the prediction performance.

We observed that almost all the proteins not exhibiting signal peptide removal had methionine excision (Figure 2(d) and Table 1). Of the 26 proteins that did not show signal peptide removal or other large $\mathrm{N}$-terminal cleavages, 24 of them began at the second amino acid. The penultimate residue was always consistent with $\mathrm{N}$-terminal methionine excision (NME): alanine, proline, threonine, serine, or glycine [27]. Given the background amino acid frequency and the expected efficiency of methionine amino peptidase [28], the binomial probability of observing such a concentration of NME matured proteins in the periplasm is 4.7 E-6. For comparison, a global top-down analysis of E. coli done recently in our lab produced a 1:1 ratio, 69 proteins without methionine excision, and 70 proteins with methionine excision (unpublished results). Additionally, a proteomic and bioinformatic analysis of NME revealed that only a minority of the proteins in a given proteome are subject to NME [29]. The functional significance for pervasive NME in the periplasm is not clear but may be related to protein stability in the potentially hazardous periplasmic environment.

Some of the identified proteins displayed large $\mathrm{N}$ terminal cleavages. For example, the uncharacterized protein Saro_1194 was observed in the data as a mature protein containing only the extreme C-terminal portion of the protein sequence, starting at residue 414, immediately after A-V-A (Figure 3). BLAST analysis showed that the annotated sequence always matched to two separate proteins, well demarcated at the $\mathrm{N}$-terminal and C-terminal extremes of the protein (Figure S1). The C-terminal portion, which was identified from the top-down MS data, also exhibited partial homology to the CHRD domain (pfam07452). Additionally, in two closely related Erythrobacter species, the two BLAST hits form a syntenic block in the genome. It is not uncommon for bacteria to combine proteins into multidomain or multifunctional proteins. However, the finding of the mature protein with a perfectly matched and cleaved signal peptide (upstream of the A-V-A is an easily detectable hydrophobic patch and basic residues) suggests that Saro_1194 is actually two separate proteins.

The bacterial periplasm is an oxidizing environment that facilitates disulfide bond formation for correct protein folding and stability $[9,10]$. Fifty-three of the 55 proteins identified using our top-down approach contained an even number of cysteines within the detected sequences (i.e., after removal of the signal peptides), including 33 proteins containing no cysteine, 17 proteins containing two cysteines, one protein containing four cysteines, and two proteins containing eight cysteines. Two proteins contain a single cysteine, phosphoribosylformylglycinamidine synthetase PurS, and the uncharacterized protein PhnA. Among the proteins containing two cysteines, only two proteins (arsenate reductase and 


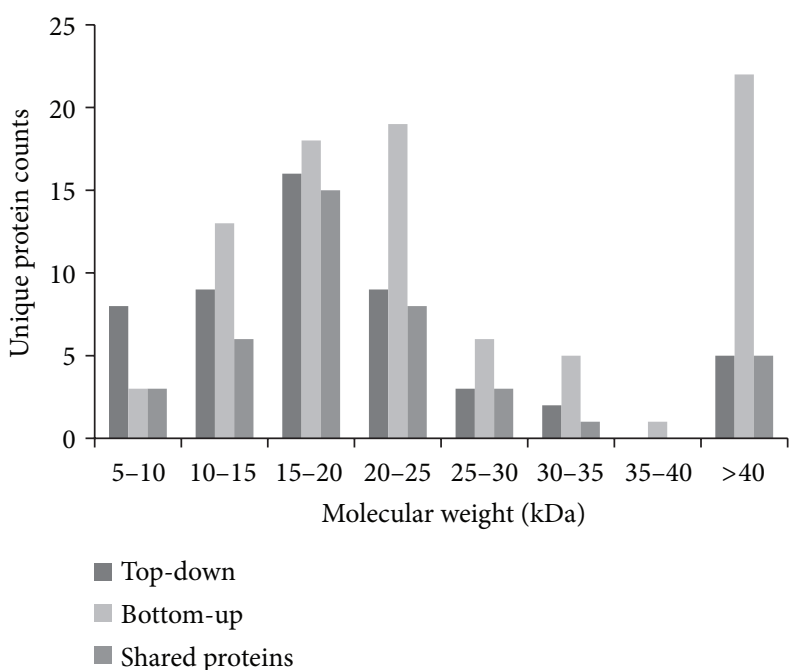

(a)

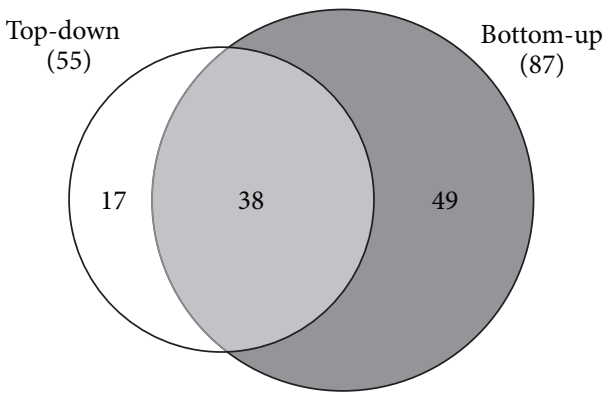

(b)

FIgURE 4: (a) Molecular mass distributions of proteins identified using top-down and bottom-up analysis. Theoretical molecular masses were calculated using amino acid sequence. (b) Overlap of proteins identified using top-down and bottom-up analysis (considering proteins identified by at least two unique peptides).

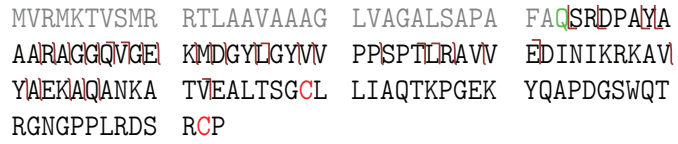

(a)

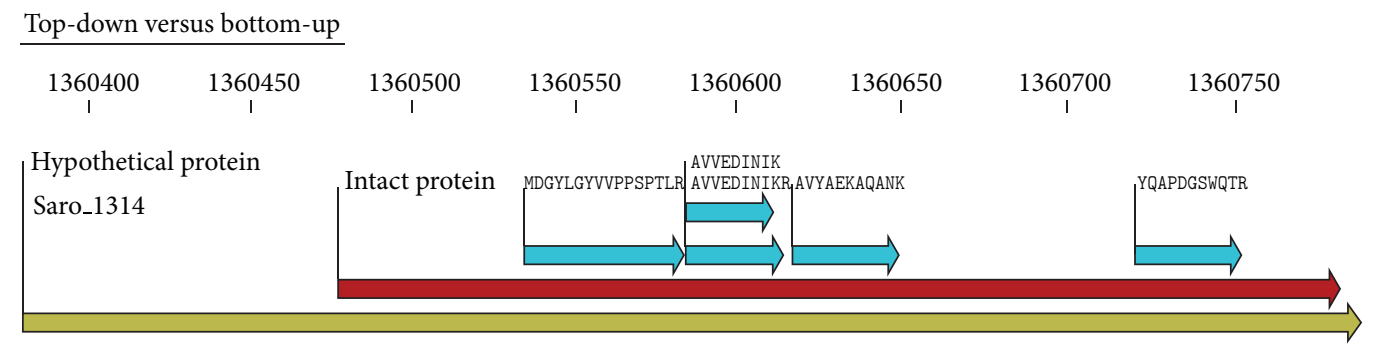

MVRMKTVSMRRTLAAVAAAGLVAGALSAPAFAQSRDPAYAAARAGGQVGEKMDGYLGYVVPPSPTLRAVVEDINIKRKAVYAEKAQANKATVEEYALTSGCLLIAQTKPGEKYQAPDGSWQTRGNGPPLRDSRCP“

(b)

FIgURE 5: Top-down and bottom-up analysis of the hypothetical protein Saro_1314. (a) Fragmentation ion map illustrating high confidence identifications ("Q" highlighted in green font was modified as pyroglutamic acid, and two "C" highlighted in red font formed a disulfide bond). (b) Sequence coverage between top-down approach and bottom-up approach (blue arrows indicate the sequences identified using bottom-up approach).

molybdopterin binding domain, Table 1) did not form a disulfide bond. Instead, both of these proteins contained a glutathionylated adduct (RSSG) on one of the cysteine residues. Although neither of these proteins was detected with signal peptide removal, it has been reported that under certain conditions, $90 \%$ of the arsenate reductase activity was found in the periplasmic faction in some bacteria (e.g., Shewanella [30]). In other bacteria, several molybdopterin binding proteins (e.g., periplasmic nitrate reductase from Desulfovibrio desulfuricans ATCC 27774) were also found in periplasmic fractions $[31,32]$. Therefore, these two proteins are likely to be periplasmic proteins, and the observation may indicate the occurrence of cysteine glutathionylation as a form of oxidation in the periplasm other than disulfide bond based oxidation.

Proteoform identifications from top-down also found other PTMs (Table 1). The most common was pyroglutamate, which was very often found on signal peptide cleaved proteins. In nine proteins where the first residue of the mature protein was glutamine, a conversion to pyroglutamate 
was observed. As mentioned earlier, two proteins were observed with S-glutathiolation, Saro_1778 molybdopterin binding domain protein and Saro_3279 arsenate reductase.

To access the sensitivity and depth of the top-down approach, the same periplasmic enriched protein fraction was analyzed by bottom-up proteomics (Supplementary Table 1 available online at http://dx.doi.org/10.1155/2013/279590). In the bottom-up analysis, 87 proteins were confidently identified with at least two unique peptides. Of these proteins, 37 were also identified in the top-down approach. Fifty proteins were detected only in the bottom-up approach, but most of them have molecular masses larger than $40 \mathrm{kDa}$ (Figure 4(a)), which makes them less amenable to top-down analysis at present. Seventeen proteins were uniquely identified in the top-down approach (Figure 4(b)); eight of these proteins have molecular masses less than $10 \mathrm{kDa}$. Characterizing small proteins represents a challenge for the bottom-up workflow due to the inability to generate sufficient tryptic peptides for analysis.

We compared the top-down and bottom-up data for their ability to detect mature protein isoforms. The bottom-up data identified 14 proteins with signal peptide cleavage, of which seven were also identified by top-down analysis. Of the remaining seven that were unique to bottom-up, four were large proteins $(>45 \mathrm{kDa})$ and thus largely inaccessible using our current top-down MS platform. We note that for 12 additional proteins, the signal peptide was identified only in the top-down approach, while peptides found in the bottomup data did not identify a signal peptide cleavage (i.e., none of the peptides captured the mature $\mathrm{N}$-terminus). For example, a hypothetical protein Saro_1314 was confidently identified with a signal peptide removal, a disulfide bond between Cys 99 and Cys 132, and an N-terminal pyroglutamate modification (Figure 5(a)). Only five tryptic peptides were detected for the same protein using the bottom-up approach, and none of them provided evidence for the PTMs (Figure 5(b)). Thus, while the bottom-up approach led to the identification of a larger number of proteins (i.e., a larger survey of periplasmic contents), the top-down analysis provided information on the mature N-terminus and other PTMs.

\section{Conclusions}

Top-down MS analysis of the intact periplasmic fraction of $N$. aromaticivorans indicated the extensive use of sec-dependent signal peptides and disulfide bond formation, as expected for a Gram-negative periplasm. Less expected was the high frequency of NME, which, to our knowledge, has not previously been reported in the bacterial periplasm. Considering these two forms of cleavage and protein maturation, almost all the proteins detected in this study were modified. Moreover, these are cleavage maturation events where no evidence was found of the unmodified protein. Although various modification types were detected, the predominant PTM observed here was proteolysis. Beyond simply showing expression of several "hypothetical" proteins, we have improved the annotation of many genes by providing localization and PTM status, which provides a basis for further functional annotation of this poorly characterized genus. We propose that top-down MS should be an integral part of efforts towards the characterization of bacterial proteomes in the future.

\section{Acknowledgments}

This research was performed in the W. R. Wiley Environmental Molecular Sciences Laboratory (EMSL, a national scientific user facility sponsored by the US Department of Energy's Office of Biological and Environmental Research and located at Pacific Northwest National Laboratory). Pacific Northwest National Laboratory is operated by Battelle Memorial Institute for the US Department of Energy under Contract DE-AC05-76RLO-1830. Portions of this work were supported by funds from EMSL intramural research projects, and EMSL capability development projects the US Department of Energy's (DOE's) Office of Biological and Environmental Research, and the NIH National Center for Research Resources (Grant RR018522). S. H. Payne was funded by an NSF Grant (EF-0949047). The authors also wish to thank Drs Xiaowen Liu and Pavel Pevzner from UCSD for providing data analysis software MS-Align+.

\section{References}

[1] J. W. Costerton, J. M. Ingram, and K. J. Cheng, "Structure and function of the cell envelope of gram negative bacteria," Bacteriological Reviews, vol. 38, no. 1, pp. 87-110, 1974.

[2] J. A. Hobot, E. Carlemalm, W. Villiger, and E. Kellenberger, "Periplasmic gel: new concept resulting from the reinvestigation of bacterial cell envelope ultrastructure by new methods," Journal of Bacteriology, vol. 160, no. 1, pp. 143-152, 1984.

[3] M. Ehrmann, The Periplasm, ASM press, Washington, DC, USA, 2006.

[4] M. Merdanovic, T. Clausen, M. Kaiser, R. Huber, and M. Ehrmann, "Protein quality control in the bacterial periplasm," Annual Review of Microbiology, vol. 65, pp. 149-168, 2011.

[5] J. W. Izard and D. A. Kendall, "Signal peptides: exquisitely designed transport promoters," Molecular Microbiology, vol. 13, no. 5, pp. 765-773, 1994.

[6] R. E. Dalbey, M. O. Lively, S. Bron, and J. M. van Dijl, "The chemistry and enzymology of the type I signal peptidases," Protein Science, vol. 6, no. 6, pp. 1129-1138, 1997.

[7] D. Missiakas and S. Raina, "Protein folding in the bacterial periplasm," Journal of Bacteriology, vol. 179, no. 8, pp. 24652471, 1997.

[8] L. M. Stancik, D. M. Stancik, B. Schmidt, D. M. Barnhart, Y. N. Yoncheva, and J. L. Slonczewski, "pH-dependent expression of periplasmic proteins and amino acid catabolism in Escherichia coli," Journal of Bacteriology, vol. 184, no. 15, pp. 4246-4258, 2002.

[9] H. Kadokura, F. Katzen, and J. Beckwith, "Protein disulfide bond formation in prokaryotes," Annual Review of Biochemistry, vol. 72, pp. 111-135, 2003.

[10] H. Kadokura and J. Beckwith, "Mechanisms of oxidative protein folding in the bacterial cell envelope," Antioxidants and Redox Signaling, vol. 13, no. 8, pp. 1231-1246, 2010.

[11] Y. Y. Londer, S. E. Giuliani, T. Peppler, and F. R. Collart, "Addressing Shewanella oneidensis "cytochromome": the first 
step towards high-throughput expression of cytochromes c," Protein Expression and Purification, vol. 62, no. 1, pp. 128-137, 2008.

[12] M. M. Savitski, M. L. Nielsen, and R. A. Zubarev, "ModifiComb, a new proteomic tool for mapping substoichiometric posttranslational modifications, finding novel types of modifications, and fingerprinting complex protein mixtures," Molecular and Cellular Proteomics, vol. 5, no. 5, pp. 935-948, 2006.

[13] S. Tanner, S. H. Payne, S. Dasari et al., "Accurate annotation of peptide modifications through unrestrictive database search," Journal of Proteome Research, vol. 7, no. 1, pp. 170-181, 2008.

[14] A. Chi, L. Valenzuela, S. Beard et al., "Periplasmic proteins of the extremophile acidithiobacillus ferrooxidans: a high throughput proteomics analysis," Molecular and Cellular Proteomics, vol. 6, no. 12, pp. 2239-2251, 2007.

[15] X. Liu, Y. Sirotkin, Y. Shen et al., "Protein identification using top-down," Molecular and Cellular Proteomics, vol. 11, no. 6, Article ID M111.008524, 2012.

[16] C. M. Ryan, P. Souda, F. Halgand et al., "Confident assignment of intact mass tags to human salivary cystatins using top-down fourier-transform ion cyclotron resonance mass spectrometry," Journal of the American Society for Mass Spectrometry, vol. 21, no. 6, pp. 908-917, 2010.

[17] O. Pinyakong, H. Habe, and T. Omori, "The unique aromatic catabolic genes in sphingomonads degrading polycyclic aromatic hydrocarbons (PAHs)," Journal of General and Applied Microbiology, vol. 49, no. 1, pp. 1-19, 2003.

[18] R. N. Brown, M. F. Romine, A. A. Schepmoes, R. D. Smith, and M. S. Lipton, "Mapping the subcellular proteome of Shewanella oneidensis MR-1 using Sarkosyl-based fractionation and LCMS/MS protein identification," Journal of Proteome Research, vol. 9, no. 9, pp. 4454-4463, 2010.

[19] R. T. Kelly, J. S. Page, Q. Luo et al., "Chemically etched open tubular and monolithic emitters for nanoelectrospray ionization mass spectrometry," Analytical Chemistry, vol. 78, no. 22, pp. 7796-7801, 2006.

[20] E. A. Livesay, K. Tang, B. K. Taylor et al., "Fully automated fourcolumn capillary LC-MS system for maximizing throughput in proteomic analyses," Analytical Chemistry, vol. 80, no. 1, pp. 294-302, 2008.

[21] S. Kim, N. Gupta, and P. A. Pevzner, "Spectral probabilities and generating functions of tandem mass spectra: a strike against decoy databases," Journal of Proteome Research, vol. 7, no. 8, pp. 3354-3363, 2008.

[22] E. Venter, R. D. Smith, and S. H. Payne, "Proteogenomic analysis of bacteria and archaea: a 46 organism case study," PLoS One, vol. 6, no. 11, article e27587, 2011.

[23] D. Perlman and H. O. Halvorson, "A putative signal peptidase recognition site and sequence in eukaryotic and prokaryotic signal peptides," Journal of Molecular Biology, vol. 167, no. 2, pp. 391-409, 1983.

[24] Y. S. Tsai, A. Scherl, J. L. Shaw et al., "Precursor ion independent algorithm for top-down shotgun proteomics," Journal of the American Society for Mass Spectrometry, vol. 20, no. 11, pp. 21542166, 2009.

[25] S. M. Patrie, J. T. Ferguson, D. E. Robinson et al., “Top down mass spectrometry of $<60-\mathrm{kDa}$ proteins from Methanosarcina acetivorans using quadrupole FTMS with automated octopole collisionally activated dissociation," Molecular and Cellular Proteomics, vol. 5, no. 1, pp. 14-25, 2006.

[26] E. J. Danoff and K. G. Fleming, "The soluble, periplasmic domain of OmpA folds as an independent unit and displays chaperone activity by reducing the self-association propensity of the unfolded OmpA transmembrane beta-barrel," Biophysical Chemistry, vol. 159, no. 1, pp. 194-204, 2011.

[27] C. Flinta, B. Persson, H. Jornvall, and G. von Heijne, "Sequence determinants of cytosolic N-terminal protein processing," European Journal of Biochemistry, vol. 154, no. 1, pp. 193-196, 1986.

[28] N. Gupta, J. Benhamida, V. Bhargava et al., "Comparative proteogenomics: combining mass spectrometry and comparative genomics to analyze multiple genomes,' Genome Research, vol. 18, no. 7, pp. 1133-1142, 2008.

[29] F. Frottin, A. Martinez, P. Peynot et al., "The proteomics of N-terminal methionine cleavage," Molecular and Cellular Proteomics, vol. 5, no. 12, pp. 2336-2349, 2006.

[30] D. Malasarn, J. R. Keeffe, and D. K. Newman, "Characterization of the arsenate respiratory reductase from Shewanella sp. strain ANA-3," Journal of Bacteriology, vol. 190, no. 1, pp. 135-142, 2008.

[31] F. Biaso, B. Burlat, and B. Guigliarelli, "DFT investigation of the molybdenum cofactor in periplasmic nitrate reductases: structure of the $\mathrm{Mo}(\mathrm{V}) \mathrm{EPR}$-active species," Inorganic Chemistry, vol. 51, no. 6, pp. 3409-3419, 2012.

[32] S. Najmudin, P. J. González, J. Trincão et al., "Periplasmic nitrate reductase revisited: a sulfur atom completes the sixth coordination of the catalytic molybdenum," Journal of Biological Inorganic Chemistry, vol. 13, no. 5, pp. 737-753, 2008. 

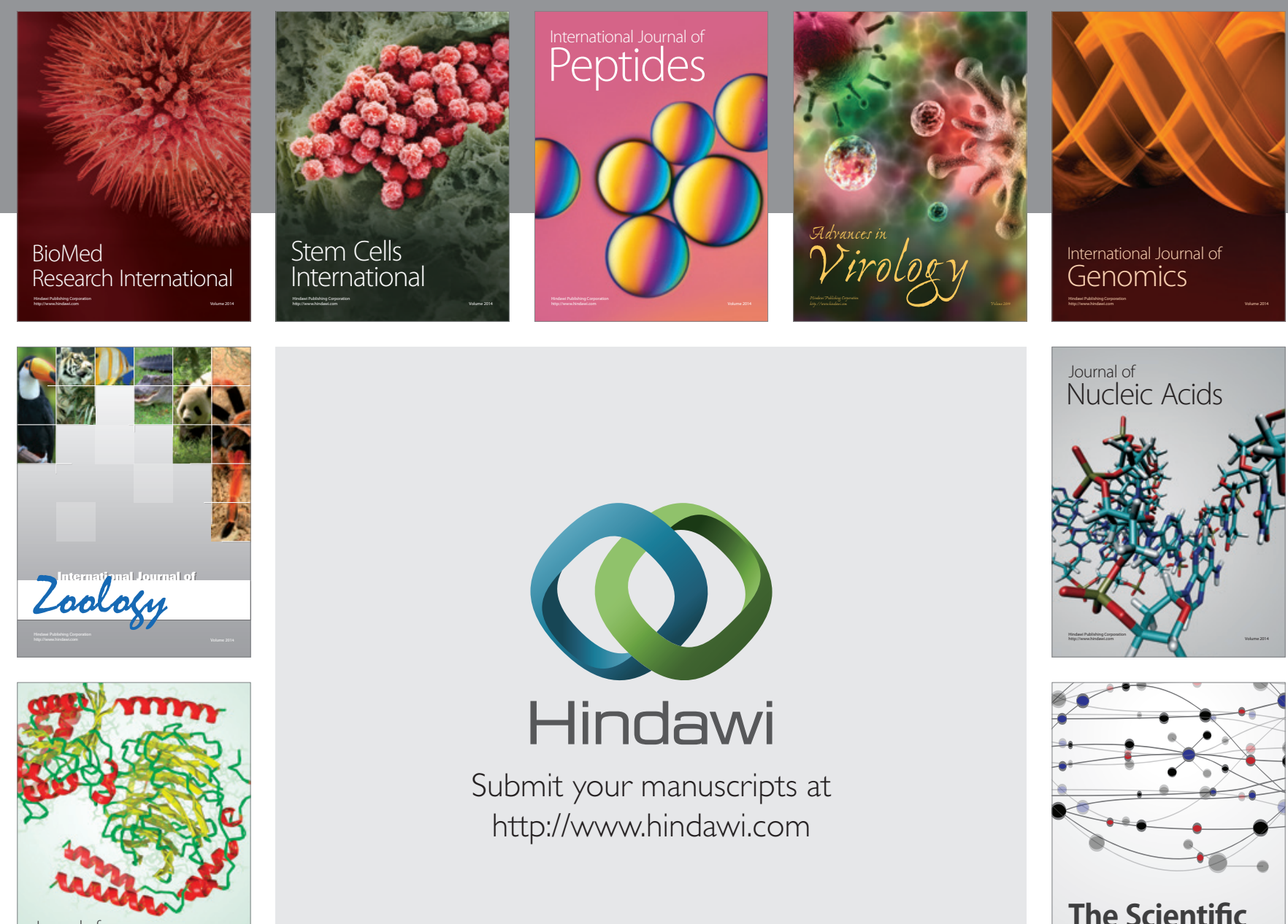

Submit your manuscripts at

http://www.hindawi.com

Journal of
Signal Transduction
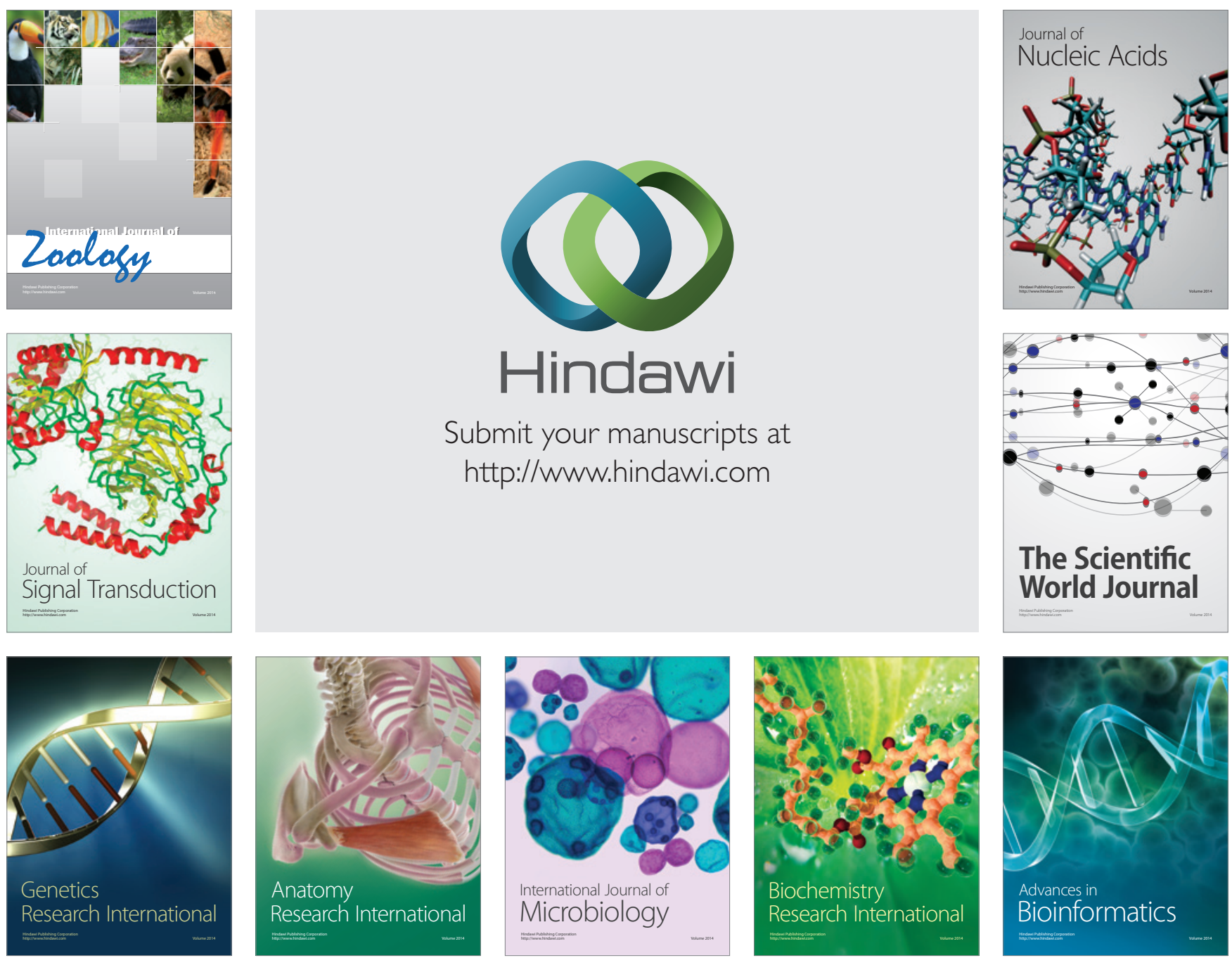

The Scientific World Journal
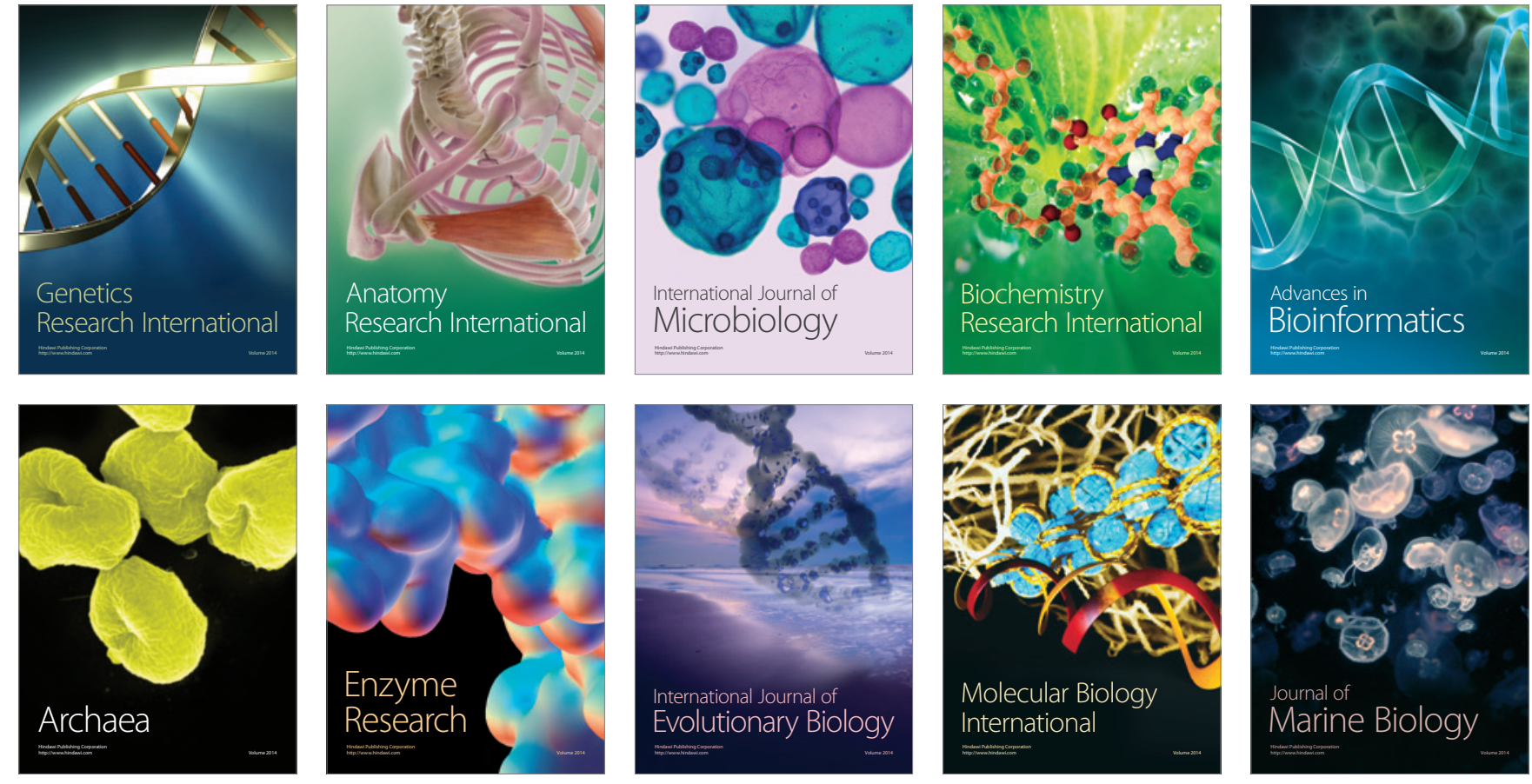